\title{
Analysis of Small-Scale Fading Distributions in Vehicle-to-Vehicle Communications
}

\author{
Vicent M. Rodrigo-Peñarrocha, ${ }^{1}$ Juan Reig, ${ }^{1}$ Lorenzo Rubio, ${ }^{1}$ \\ Herman Fernández, ${ }^{2}$ and Susana Loredo ${ }^{3}$ \\ ${ }^{1}$ Electromagnetic Radiation Group (GRE), Universitat Politècnica de València, Camino de Vera, s/n, 46022 Valencia, Spain \\ ${ }^{2}$ Escuela de Ingeniería Electrónica, Universidad Pedagógica y Tecnológica de Colombia, Sogamoso, Colombia \\ ${ }^{3}$ Electrical Engineering Department, University of Oviedo, 33204 Gijón, Spain
}

Correspondence should be addressed to Juan Reig; jreigp@dcom.upv.es

Received 21 December 2015; Revised 8 June 2016; Accepted 28 June 2016

Academic Editor: Yuh-Shyan Chen

Copyright (C) 2016 Vicent M. Rodrigo-Peñarrocha et al. This is an open access article distributed under the Creative Commons Attribution License, which permits unrestricted use, distribution, and reproduction in any medium, provided the original work is properly cited.

\begin{abstract}
This work analyzes the characteristics of the small-scale fading distribution in vehicle-to-vehicle (V2V) channels. The analysis is based on a narrowband channel measurements campaign at $5.9 \mathrm{GHz}$ designed specifically for that purpose. The measurements were carried out in highway and urban environments around the city of Valencia, Spain. The experimental distribution of the small-scale fading is compared to several analytical distributions traditionally used to model the fast fading in wireless communications, such as Rayleigh, Nakagami- $m$, Weibull, Rice, and $\alpha-\mu$ distributions. The parameters of the distributions are derived through statistical inference techniques and their goodness-of-fit is evaluated using the Kolmogorov-Smirnov (K-S) test. Our results show that the $\alpha-\mu$ distribution exhibits a better fit compared to the other distributions, making its use interesting to model the small-scale fading in V2V channels.
\end{abstract}

\section{Introduction}

Over the past ten years, the vehicular propagation channel has attracted the interest of numerous researchers provided that new frequency bands have been allocated for vehicular systems [1-4]. Nevertheless, the vehicle-to-vehicle (V2V) communications channel presents special peculiarities with regard to the fixed-to-mobile (F2M) scenarios, that is, the considerable mobility of both the transmitter $(\mathrm{Tx})$ and receiver $(\mathrm{Rx})$ terminals and the interacting objects such as reflectors and/or scatterers $[1,5]$. In addition, the low elevation of the antennas determines a significant obstruction probability of $\mathrm{Tx}-\mathrm{Rx}$ links, particularly in urban environments with dense road traffic. Also, the propagation mechanisms in the dedicated short-range communications (DSRC) band at $5.9 \mathrm{GHz}$ are very different from those occurring in the traditional cellular communications bands, that is, from 1 to $2 \mathrm{GHz}$. Therefore, as a part of the narrowband channel characterization, accurate models for the small-scale fading distribution in $\mathrm{V} 2 \mathrm{~V}$ propagation channels are essential to develop, evaluate, and validate new protocols and system architecture configurations [6,7].

The characterization of the small-scale fading distribution in V2V communications has been analyzed in different environments [8-15]. In [8] the Rice distribution turned out to be the best-fit distribution at $5.2 \mathrm{GHz}$ in urban, suburban, motorway, and highway scenarios by approximating the cumulative distribution functions (CDFs) with the leastsquare (LS) algorithm. Also, in a more recent paper [9], the Rice distribution is used to model the envelope of the first delay bin in $\mathrm{V} 2 \mathrm{~V}$ channels. The Weibull distribution parameters were extracted from campus, highway, suburban, and urban areas at $5.3 \mathrm{GHz}$ in [10] by adjusting the Weibull CDF to the experimental CDF with the mean-square-error (MSE) method. In [11] a combined maximum-likelihood estimator (MLE) and matching the CDF method was used to obtain the parameters of the Nakagami- $m$ distribution at $5.9 \mathrm{GHz}$ in a highway environment. The fading parameter, $m$, of the Nakagami- $m$ was estimated using the MLE procedure at $5.9 \mathrm{GHz}$ in a suburban environment in [12]. In this work, 
the dependence of $m$ on the Tx and Rx separtation distance and the goodness-of-fit using the Kolmogorov-Smirnov (K$S)$ test were performed over the estimated Nakagami- $m$ distributions. Recently, in [13] the Rayleigh, Rice, Nakagami$m$, and Weibull distributions parameters have been estimated using the MLE method at $5.805 \mathrm{GHz}$ with a large object (bus) located between Tx and Rx. The goodness-of-fit of the analytical estimated distributions in [13] has been checked by using both the Akaike information criteria (AIC) and the K-S tests. In [14], the authors found Weibull scale factors lower than 2 in the $5 \mathrm{GHz}$ band, which correspond to more severe conditions than the Rayleigh fading. This fact is explained by the existence of measurement records with transitions between line-of-sight (LOS) and non-LOS (NLOS) conditions [16].

A few years ago, the $\alpha-\mu$ distribution was proposed to model the small-scale fading amplitude in inhomogeneous scattering field environments [17]. Nevertheless, to the best of the authors' knowledge, the $\alpha-\mu$ distribution has not been used to estimate the small-scale distribution in V2V communications yet. This paper estimates the parameters of the Rayleigh, Rice, Weibull, Nakagami- $m$, and $\alpha-\mu$ distributions to analyze the small-scale fading in $\mathrm{V} 2 \mathrm{~V}$ from a narrowband channel measurements campaign designed specifically for that purpose. The parameters of the distributions considered have been estimated by statistical inference. The results derived from the application of the K-S statistical test have shown that the Nakagami- $m$, Weibull, Rice, and $\alpha-\mu$ distributions can match satisfactorily the experimental (empirical) distribution. As a novelty, our analysis shows that the $\alpha-\mu$ distribution exhibits a better fit compared to the other distributions, making its use interesting to model the smallscale fading in $\mathrm{V} 2 \mathrm{~V}$ channels, where it is difficult to separate both the small- and large-scale fading effects as a consequence of the large channel variations.

\section{Measurement Campaign}

2.1. Measurement Setup. In order to characterize the V2V channel, a specific narrowband channel sounder has been designed. The HP83623A signal generator (SG) was used at the Tx side. The SG transmitted a continuous wave at $5.9 \mathrm{GHz}$. A high power amplifier (HPA) enables transmission with $+23.8 \mathrm{dBm}$ of equivalent isotropic radiated power (EIRP). The ZVA24 Rohde \& Schwarz vector network analyzer (VNA), configured as a power meter $(0 \mathrm{~Hz}$ of SPAN and a central frequency equal to $5.9 \mathrm{GHz}$ ), was used to measure the received power level directly through the $b_{2}$ parameter at $\mathrm{Rx}$. The $b_{2}$ parameter was continuously measured using traces of 5000 test points. The VNA was configured with a resolution bandwidth of the Intermediate Filter $\left(B_{\mathrm{IF}}\right)$ equal to $100 \mathrm{kHz}$ and a sweep time per trace of $2 \mathrm{~s}$, resulting in a sampling time (acquisition time per test point) of about $1 \mathrm{~ms}$. A laptop controlled the VNA to automate the measurement acquisition system.

Both $\mathrm{Tx}$ and $\mathrm{Rx}$ used the same antenna, a $\lambda / 4$ monopole. The antenna gain was measured in an anechoic chamber, mounting the antenna over a $1 \times 1-\mathrm{m}^{2}$ metallic plane emulating the roof of a car. The value of the antenna

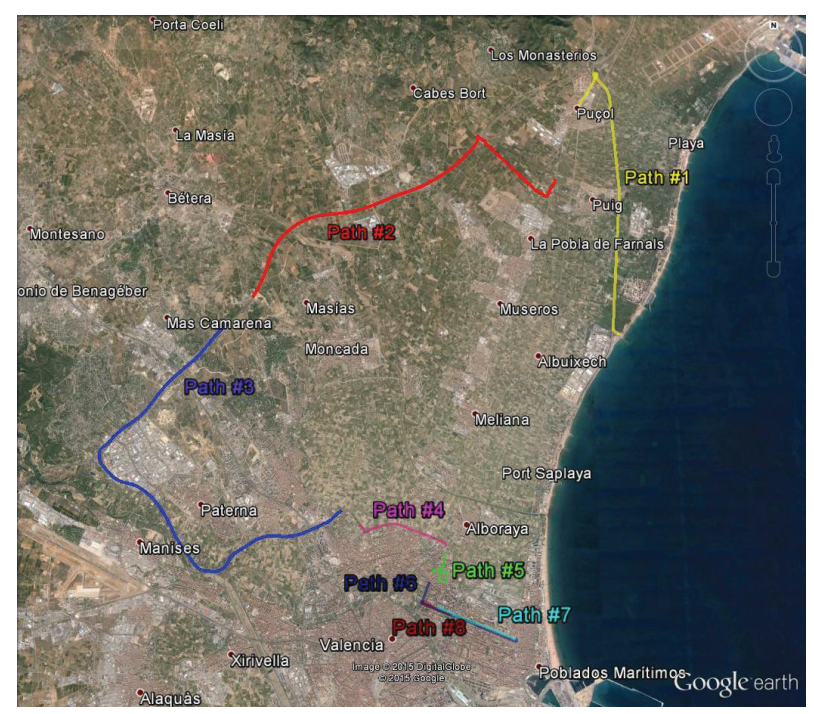

FIgURE 1: Paths driven in the measurement campaign.

gain measured in the horizontal plane was approximately $-2.56 \mathrm{~dB}$. In addition, both $\mathrm{Tx}$ and $\mathrm{Rx}$ systems were equipped with GPSs (Global Positioning Systems), each one controlled by a laptop, to provide continuous information about the acquisition measurement time, as well as the relative speed between $\mathrm{Tx}$ and $\mathrm{Rx}$, and their separation distance. The laptops were synchronized to relate the data from the different GPSs and the measurements from the VNA. More details about the measurement setup, but for a different VNA configuration, can be found in [7].

\subsection{Measurement Environment. A total of 8 routes were} measured in different environments around the city of Valencia, Spain. A map describing all the routes is depicted in Figure 1, and two photos of highway and urban environments, respectively, are illustrated in Figure 2. Also, more details about the measurements are presented in Table 1. Routes \#1\#3 correspond to highway with heavy road traffic density and two or three lanes in each direction. Both sides of the highways are open areas. Routes \#4-\#8 are urban streets with several conditions. Route \#5 corresponds to narrow streets of the city of Valencia with one lane, whereas routes \#4 and \#6$\# 8$ are wide streets with at least three lanes in each direction.

The total driven distance for each route and the maximum Tx-Rx separation distance are shown in Table 1. It is worth noting that both Tx and Rx were moving in convoy, that is, in the same direction, during the measurement acquisition. The maximum $T x-R x$ separation between both vehicles ranged from $72.2 \mathrm{~m}$ (in route \#5) to $440.53 \mathrm{~m}$ (in route \#1).

The absolute value of the relative speed between Tx and $\mathrm{Rx}, v_{\text {rel }}$, can be defined as

$$
v_{\text {rel }}=\left|v_{\mathrm{Tx}}-v_{\mathrm{Rx}}\right|,
$$

where $|\cdot|$ represents the absolute value and $v_{\mathrm{Tx}}$ and $v_{\mathrm{Rx}}$ are the Tx and Rx speeds, respectively.

In order to analyze the small-scale fading statistics, each measurement record of each route has been divided in 


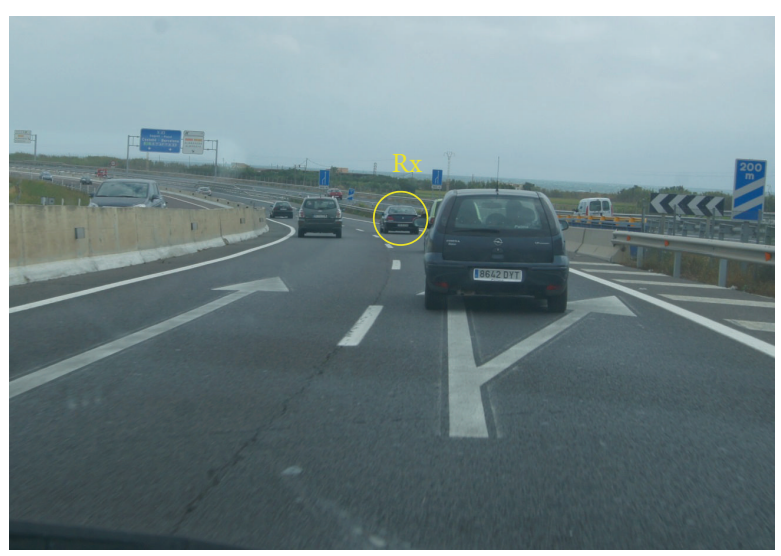

(a)

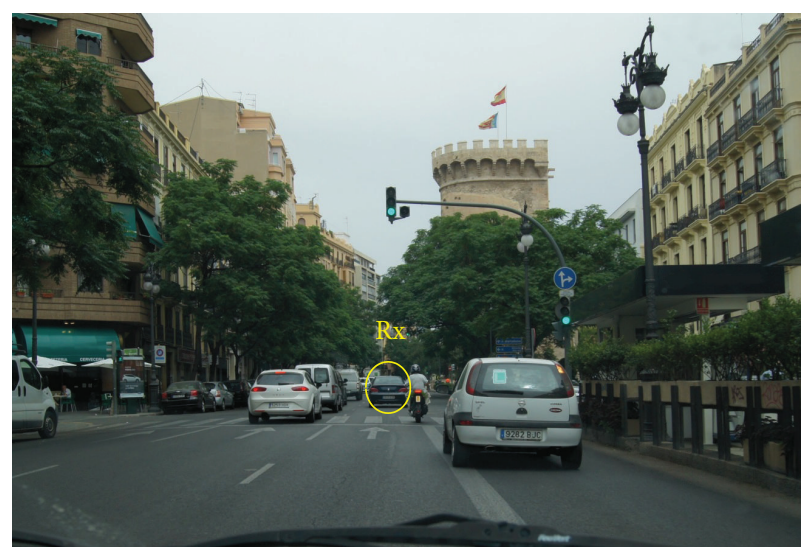

(b)

Figure 2: Photos of (a) highway and (b) urban environments in the measurement campaign.

TABLE 1: Main parameters of each route.

\begin{tabular}{|c|c|c|c|c|}
\hline Parameters & $\begin{array}{l}\text { Driven } \\
\text { distance }\end{array}$ & $\begin{array}{c}\text { Power } \\
\text { mean } \\
\text { standard } \\
\text { deviation }\end{array}$ & $\begin{array}{l}\text { Maximum } \\
\text { Tx-Rx } \\
\text { separation }\end{array}$ & $\begin{array}{c}\text { Maximum } \\
\text { relative } \\
\text { speed }\end{array}$ \\
\hline Units & $\mathrm{km}$ & $\mathrm{dB}$ & $\mathrm{m}$ & $\mathrm{km} / \mathrm{h}$ \\
\hline \multicolumn{5}{|l|}{ Route \#1 } \\
\hline Highway & 10.762 & 2.17 & 440.53 & 49.14 \\
\hline \multicolumn{5}{|l|}{ Route \#2 } \\
\hline Highway & 13.547 & 1.91 & 176.14 & 26.74 \\
\hline \multicolumn{5}{|l|}{ Route \#3 } \\
\hline Highway & 15.089 & 1.90 & 243.90 & 35.90 \\
\hline \multicolumn{5}{|l|}{ Route \#4 } \\
\hline Urban & 2.979 & 3.89 & 122.35 & 31.46 \\
\hline \multicolumn{5}{|l|}{ Route \#5 } \\
\hline Urban & 1.929 & 3.57 & 72.20 & 30.13 \\
\hline \multicolumn{5}{|l|}{ Route \#6 } \\
\hline Urban & 3.654 & 2.57 & 148.15 & 28.13 \\
\hline \multicolumn{5}{|l|}{ Route \#7 } \\
\hline Urban & 2.461 & 3.61 & 112.61 & 32.06 \\
\hline \multicolumn{5}{|l|}{ Route \#8 } \\
\hline Urban & 1.162 & 3.54 & 173.95 & 36.42 \\
\hline
\end{tabular}

windows of $100 \lambda$, which correspond to a variable window size in number of samples due to the variation of the receiver vehicle speed with an almost constant sample period of around $1 \mathrm{~ms}$.

The mean standard deviation of the received power in each $100 \lambda$-sized window provides a measure of the signal dispersion. From Table 1, all the routes present small dispersion, specially the highway routes, provided that the theoretical maximum standard deviation is $5.57 \mathrm{~dB}$ which corresponds to the Rayleigh distribution. It should be noted that only $4.78 \%$ of all the $100 \lambda$-sized windows exceeded $5.57 \mathrm{~dB}$. Such a low mean standard deviation of the received power reflects a dominant multipath component (MPC) in most of the extracted windows.

\section{Results}

In this section, the parameters of the Nakagami- $m$, Weibull, Rice, and $\alpha-\mu$ distributions are estimated. The goodness-offit of these distributions is calculated by using the K-S test [18]. Then, the assessment of the matching of the previous distributions to the experimental distribution is carried out for the lower tails of the distribution, using the difference between the experimental and estimated CDFs.

3.1. Parameters of the Estimated Distributions. The parameters of the Rayleigh, Nakagami- $m$, Weibull, Rice, and $\alpha-\mu$ distributions have been estimated over each window of each route: the fading parameter, $m$, for the Nakagami- $m$ distribution; the shape parameter, $\alpha$, for the Weibull distribution; the $K$-parameter for the Rice distribution; and the $\alpha$ and $\mu$ parameters for the $\alpha-\mu$ distribution, defined in [17]. For further details about the estimators of such distributions, the reader is addressed to [19]. Since all the distributions of these parameters are considerably right-skewed, we have derived the distribution for each parameter in logarithmic units, that is, $10 \log p$, where $p$ is the parameter analyzed.

The mean standard deviation, the skewness, and the kurtosis $[20,21]$ of the parameters of such distributions in logarithmic units are summarized in Table 2. The skewness is an estimation of the asymmetry of the probability distribution and it is defined as

$$
\widehat{\gamma}_{1} \triangleq \frac{\widehat{\mu}_{3}}{\widehat{\mu}_{2}^{3 / 2}},
$$

where $\widehat{\mu}_{n}$ is the $n$th sample central moment of the distribution. If $\widehat{\gamma}_{1}=0$ the distribution is symmetrical. Values of right-skewed distributions provide $\widehat{\gamma}_{1}>0$, and $\widehat{\gamma}_{1}<0$ corresponds to left-skewed distributions. It is worth noting that the distributions used to model the small-scale fading due to the multipath effect are asymmetrical. 
TABLE 2: Parameters of the distributions in logarithmic units.

\begin{tabular}{|c|c|c|c|c|c|}
\hline \multirow{2}{*}{$\begin{array}{l}\text { Distribution } \\
\text { Parameter }\end{array}$} & \multirow{2}{*}{$\begin{array}{c}\text { Nakagami- } m \\
m\end{array}$} & \multirow{2}{*}{$\begin{array}{c}\text { Weibull } \\
\alpha\end{array}$} & \multirow{2}{*}{$\begin{array}{c}\text { Rice } \\
K\end{array}$} & \multicolumn{2}{|c|}{$\alpha-\mu$} \\
\hline & & & & $\alpha$ & $\mu$ \\
\hline \multicolumn{6}{|l|}{$\begin{array}{l}\text { Total } \\
\text { measurements }\end{array}$} \\
\hline Mean $(\mathrm{dB})$ & 7.75 & 7.5 & 10 & 5.38 & 4.25 \\
\hline Std. dev. (dB) & 5.82 & 3.02 & 6.31 & 5.26 & 8.74 \\
\hline Skewness & 0.53 & 0.2 & -0.16 & 0.84 & 0.98 \\
\hline Kurtosis & 2.59 & 2.21 & 2.78 & 6.29 & 6.14 \\
\hline \multicolumn{6}{|l|}{ Route \#1 } \\
\hline Mean (dB) & 8.54 & 7.96 & 10.9 & 5.48 & 4.92 \\
\hline Std. dev. (dB) & 5.38 & 2.75 & 5.57 & 5.51 & 9.08 \\
\hline Skewness & 0.37 & 0.03 & -0.17 & 0.65 & 0.91 \\
\hline Kurtosis & 2.58 & 2.35 & 2.84 & 5.98 & 6.66 \\
\hline \multicolumn{6}{|l|}{ Route \#2 } \\
\hline Mean $(\mathrm{dB})$ & 10.32 & 8.8 & 12.61 & 5.82 & 5.85 \\
\hline Std. dev. (dB) & 6.27 & 3.12 & 6.29 & 6.13 & 9.63 \\
\hline Skewness & 0.17 & -0.16 & -0.43 & 0.91 & 0.61 \\
\hline Kurtosis & 2.37 & 2.35 & 3.13 & 5.02 & 4.82 \\
\hline \multicolumn{6}{|l|}{ Route \#3 } \\
\hline Mean $(\mathrm{dB})$ & 7.68 & 7.46 & 10.01 & 5.21 & 4.52 \\
\hline Std. dev. (dB) & 5.72 & 3 & 6.18 & 5.42 & 8.97 \\
\hline Skewness & 0.37 & -0.16 & -0.22 & 0.72 & 0.9 \\
\hline Kurtosis & 2.26 & 2.35 & 2.54 & 5.68 & 5.5 \\
\hline \multicolumn{6}{|l|}{ Route \#4 } \\
\hline Mean $(\mathrm{dB})$ & 2.85 & 4.88 & 4.21 & 5.17 & 0.11 \\
\hline Std. dev. (dB) & 2.86 & 1.72 & 4.55 & 3.14 & 5.02 \\
\hline Skewness & 1.31 & 0.91 & -0.14 & 0.86 & 0.59 \\
\hline Kurtosis & 5.33 & 3.63 & 3.13 & 4.48 & 5.23 \\
\hline \multicolumn{6}{|l|}{ Route \#5 } \\
\hline Mean $(\mathrm{dB})$ & 3.27 & 5.18 & 5.26 & 5.59 & -0.05 \\
\hline Std. dev. (dB) & 2.39 & 1.47 & 3.59 & 3.31 & 5.05 \\
\hline Skewness & 0.62 & 0.34 & -0.56 & 0.15 & 1.08 \\
\hline Kurtosis & 3.07 & 2.62 & 3.71 & 6.19 & 10.71 \\
\hline \multicolumn{6}{|l|}{ Route \#6 } \\
\hline Mean (dB) & 6.59 & 7 & 8.82 & 4.83 & 3.98 \\
\hline Std. dev. (dB) & 4.19 & 2.32 & 5.43 & 4.01 & 7.42 \\
\hline Skewness & 0.13 & -0.19 & -1.03 & 0.17 & 1.68 \\
\hline Kurtosis & 2.39 & 2.28 & 4.45 & 9.79 & 10.81 \\
\hline \multicolumn{6}{|l|}{ Route \#7 } \\
\hline Mean $(\mathrm{dB})$ & 3.72 & 5.36 & 5.35 & 4.92 & 1.15 \\
\hline Std. dev. (dB) & 3.67 & 2.13 & 5.2 & 2.93 & 5.27 \\
\hline Skewness & 0.96 & 0.72 & -0.05 & 1.06 & 0.7 \\
\hline Kurtosis & 3.15 & 2.5 & 2.58 & 7.75 & 5.15 \\
\hline \multicolumn{6}{|l|}{ Route \#8 } \\
\hline Mean $(\mathrm{dB})$ & 3.78 & 5.42 & 6.07 & 4.85 & 1.3 \\
\hline Std. dev. (dB) & 3.23 & 1.99 & 5.11 & 3.26 & 5.11 \\
\hline Skewness & 0.11 & -0.03 & -1.14 & 0.78 & 1.15 \\
\hline Kurtosis & 1.65 & 1.57 & 3.78 & 6.77 & 11.87 \\
\hline
\end{tabular}

The kurtosis is a measure of the "tailedness" of a probability distribution. Higher kurtosis involves a larger concentration of data around the average distribution while coexisting with a relatively high frequency of data far from the average of the data. The kurtosis is defined as

$$
\widehat{\beta}_{2} \triangleq \frac{\widehat{\mu}_{4}}{\widehat{\mu}_{2}^{2}} .
$$

Note that the Gaussian distribution, used to model the largescale fading or shadowing effect, exhibits $\widehat{\gamma}_{1}=0$ and $\widehat{\beta}_{2}=3$.

Analyzing Table 2, we first stop at standard deviation. In all routes, the Weibull $\alpha$ parameter, whose standard deviation ranges from 1.47 to $3.12 \mathrm{~dB}$, presents, in each route analyzed, the lowest standard deviation of all distribution parameters. This behaviour is due to the fact that, in many cases, the Weibull distribution provides the best match for the lower tails of the experimental distribution. Also, all the estimated distributions are significantly symmetrical with a maximum absolute value of the skewness of 1.68 for the $\mu$ parameter of the $\alpha-\mu$ distribution. Furthermore, the mean of the Rician $K$-parameter is significantly high (larger than $10 \mathrm{~dB}$ ) for the highway environment routes. In the same way, the estimated means of the fading parameter, $m$, of the Nakagami- $m$ distribution reach high values for routes \#1-\#3, ranging from 7.68 to $10.32 \mathrm{~dB}$. These facts suggest a dominant MPC prevailing in the highway environment. In contrast, moderate means of the Rician $K$-parameter have been obtained for urban environments, ranging from 4.21 to $8.82 \mathrm{~dB}$. Also, low means of the Nakagami- $m$ fading parameter have been found in urban environments, ranging from 2.85 to $6.59 \mathrm{~dB}$ (2.12 to 4.56 in linear units). The standard deviations of the estimated fading parameters are substantially smaller in urban than in highway environments, with a maximum value of the standard deviation of the fading parameter of $4.19 \mathrm{~dB}$ and $6.27 \mathrm{~dB}$ in the highway and urban routes, respectively.

On the other hand, the smallest values of the standard deviation correspond to the $\alpha$ parameter for the Weibull distribution with a maximum of $3.12 \mathrm{~dB}$ in route \#2.

The dependence of the estimated Nakagami- $m$ fading parameter, $m$, on the $\mathrm{Tx}-\mathrm{Rx}$ separation distance has been also analyzed by using a boxplot graph. Figure 3 shows the mean and the dispersion of the estimated fading parameter as a function of the distance for all the $100 \lambda$-sized windows corresponding to (a) the total measurement record and (b) route \#6. In these boxplots, the fading parameter is categorized by intervals of separations between Tx and Rx, where the central line in red is the median, the edges of the box are the 25th and 75th percentiles, and the whiskers extend to the most extreme datapoints that the algorithm considers to be not outliers. No dependence of the median of the estimated $m$ parameter on the Tx-Rx separation can be inferred from this graph. Likewise, we have not found any dependence in the median of the fading parameter on the separation for each route except for route \#6, which holds a decreasing value with the distance as it can be shown in Figure 3. The decreasing trend in the median of the fading parameter for route \#6 is in accordance with the results of [12] for suburban driving conditions. 


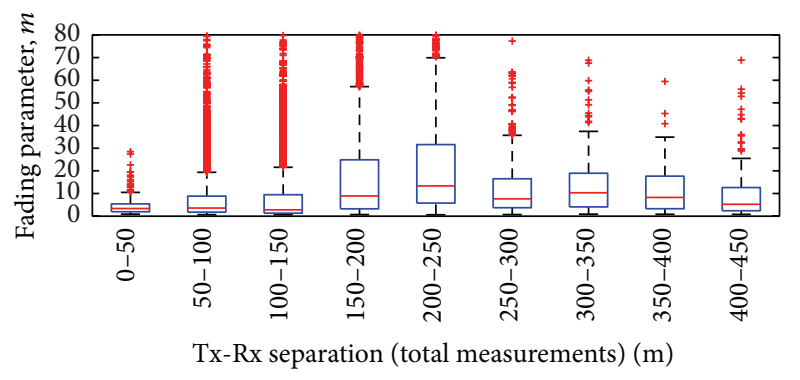

(a)

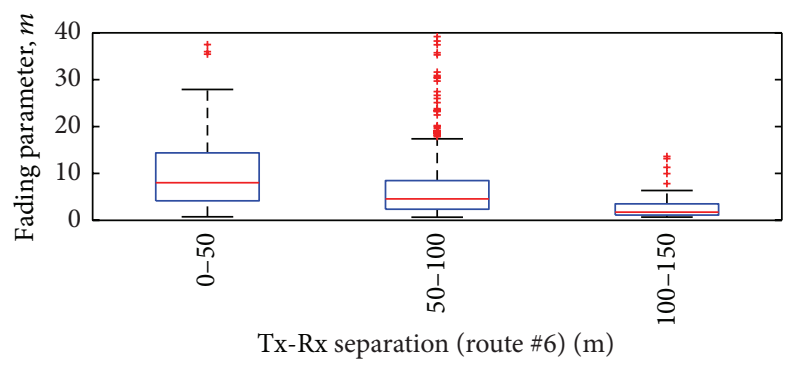

(b)

FIGURE 3: Boxplots of the fading parameter, $m$, of the Nakagami- $m$ distribution as a function of the distance between transmitter and receiver for (a) the total measurement record and (b) route \#6.

3.2. K-S Test. Once the estimated parameters of the Rayleigh, Nakagami- $m$, Weibull, Rice, and $\alpha-\mu$ distributions were calculated, we have compared such distributions to the experimental distribution in each window.

The K-S test in one given extracted window is fulfilled if the maximum absolute value between the experimental and the estimated CDFs, $D_{\text {estimated }}$, is lower than or equal to a given value, $k_{n}(p)$, which depends on both the number of samples, $n$, and the significance level, $p$. Therefore, the condition to be accomplished in each window is as follows:

$$
\begin{aligned}
D_{\text {estimated }} & =\max _{i=1, \ldots, n}\left|\widehat{F}_{\text {estimated }}\left(x_{i}\right)-F_{\text {experimental }}\left(x_{i}\right)\right| \\
& \leq k_{n}(p),
\end{aligned}
$$

where $\widehat{F}_{\text {estimated }}\left(x_{i}\right)$ is the CDF of the estimated distribution, $F_{\text {experimental }}\left(x_{i}\right)$ is the CDF of the experimental (empirical) distribution, and

$$
k_{n}(p)= \begin{cases}\frac{1.63}{\sqrt{n}}, & p=1 \% \\ \frac{1.36}{\sqrt{n}}, & p=5 \%\end{cases}
$$

with $n>50$.

The K-S statistical test has been applied to the Rayleigh, Nakagami- $m$, Weibull, Rice, and $\alpha-\mu$ distributions over 2112 windows of size $100 \lambda$ with a significance level of $5 \%$. The results are summarized in Table 3. In addition, Table 3 includes the percentage of windows in which each distribution matches the most experimental measurements denoted as best-fit (\%), that is, the lowest value given by (4) with respect to the remainder of the distributions analyzed. Note that the sum of all the percentages corresponding to the best-fit distributions is equal to $100 \%$ for each route or the total data analyzed. The distributions with the highest bestfit percentages for each route and for the total measurement records are highlighted in bold letters. From Table 3, the distributions Nakagami- $m$, Weibull, Rice, and $\alpha-\mu$ match satisfactorily the experimental distribution with fulfillment percentages of the K-S test higher than $78 \%$ for a significance level of $5 \%$ in the total measurement record. Such percentages exceed $72.49 \%$ in all the routes except route \#5. Lower fulfillment percentages in route \#5 suggest a faster variation of the
TABLE 3: K-S test fulfillment percentage for a confidence interval of $5 \%$ and best-fit distributions percentage.

\begin{tabular}{lccccc}
\hline Distributions & Rayleigh & Nakagami- $m$ & Weibull & Rice & $\alpha-\mu$ \\
\hline $\begin{array}{l}\text { Total measurements } \\
\text { K-S 5\% }\end{array}$ & 8.47 & 80.12 & 80.02 & 82.33 & 80.93 \\
Best-fit (\%) & 1.26 & 12.83 & 25.97 & 14.32 & $\mathbf{4 5 . 6 2}$ \\
\hline Route \#1 & & & & & \\
K-S 5\% & 4.50 & 80.82 & 78.27 & 81.77 & 82.00 \\
Best-fit (\%) & 0.66 & 14.63 & 24.72 & 13.68 & $\mathbf{4 6 . 3 1}$ \\
\hline Route \#2 & & & & & \\
K-S 5\% & 4.77 & 84.16 & 80.10 & 84.54 & 80.85 \\
Best-fit (\%) & 0.71 & 13.84 & 21.41 & 13.33 & $\mathbf{5 0 . 7 1}$ \\
\hline Route \#3 & & & & & \\
K-S 5\% & 9.01 & 80.41 & 80.55 & 79.5 & 80.48 \\
Best-fit (\%) & 1.76 & 13.77 & 28.57 & 12.74 & $\mathbf{4 3 . 1 6}$ \\
\hline Route \#4 & & & & & \\
K-S 5\% & 24.4 & 78.5 & 89.93 & 89.25 & 82.59 \\
Best-fit (\%) & 2.22 & 7.17 & 28.50 & 21.67 & $\mathbf{4 0 . 4 4}$ \\
\hline Route \#5 & & & & & \\
K-S 5\% & 6.05 & 49.21 & 63.95 & 61.58 & 64.21 \\
Best-fit (\%) & 0.79 & 9.21 & 35.53 & 17.36 & $\mathbf{3 7 . 1 1}$ \\
\hline Route \#6 & & & & & \\
K-S 5\% & 9.89 & 83.57 & 78.13 & 88.16 & 84.82 \\
Best-fit (\%) & 1.67 & 11.14 & 23.96 & 15.32 & $\mathbf{4 7 . 9 1}$ \\
\hline Route \#7 & & & & & \\
K-S 5\% & 19.83 & 77.89 & 86.15 & 89.46 & 84.71 \\
Best-fit (\%) & 1.65 & 8.88 & 31.40 & 18.40 & $\mathbf{3 9 . 6 7}$ \\
\hline Route \#8 & & & & & \\
K-S 5\% & 15.72 & 72.49 & 82.53 & 81.66 & 81.22 \\
Best-fit (\%) & 3.06 & 6.11 & 29.26 & 17.03 & $\mathbf{4 4 . 5 4}$ \\
\hline
\end{tabular}

propagation conditions over each window in narrow streets compared to wide streets or highways. The $\alpha-\mu$ distribution exhibits the smallest values of $D_{\text {estimated }}$ in $45.62 \%$ of the total windows analyzed. 


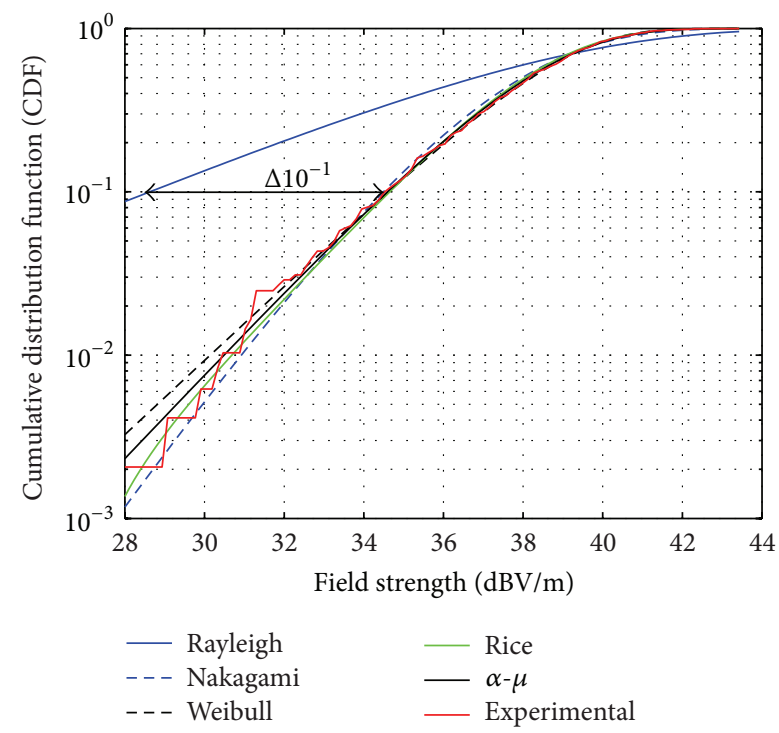

Figure 4: Cumulative distribution of the field strength in one window of route \#7. The difference between the field strength of the estimated CDF and the field strength of the experimental CDF for $P=10^{-1}$ is depicted for the Rayleigh distribution.

3.3. Lower Tails of the Estimated Distributions. The matching in the lower tails of the estimated to the experimental distributions affects significantly the wireless performance parameters, such as the bit error rate (BER) and the outage probability [22].

Therefore, in addition to the K-S test we need to assess the behaviour of the distribution specifically in the lower tails by using a procedure which evaluates the mismatch between the estimated and the experimental CDFs.

Let $\Delta_{P}$ be the difference between the received field strength of the estimated CDF and the field strength of the experimental CDF for the same value of the CDF, $P$, defined as

$$
\Delta_{P}=\left(x_{\text {estimated }}-x_{\text {experimental }}\right)_{P},
$$

where $x_{\text {estimated }}$ and $x_{\text {experimental }}$ are the values of field strength which provide $\widehat{F}_{\text {estimated }}\left(x_{\text {estimated }}\right)=P$ and $F_{\text {experimental }}\left(x_{\text {experimental }}\right)=P$, respectively.

Figure 4 shows the CDF in terms of the field strength, where the definition of $\Delta_{P}$ is illustrated. In this figure, the value of $\Delta_{P}$, with $P=10^{-1}$, for the Rayleigh distribution is indicated for one window of route \#7.

Table 4 summarizes the mean and the root-meansquare (rms) values for $\Delta_{P=10^{-1}}$ of the estimated Rayleigh, Nakagami- $m$, Weibull, Rice, and $\alpha-\mu$ distributions in each route and in the total measurement record. The distributions with smallest rms values are highlighted in bold letters. The values of $\Delta_{P=10^{-1}}$ are low biased (small absolute values of the mean) except for the Rayleigh distribution. Also, the Weibull distribution provides the smallest rms of $\Delta_{P=10^{-1}}$ for the whole measurement windows and particularly for each route except routes \#2 and \#5.
TABLE 4: Mean and root-mean-square (rms) of the difference between the field strength of the estimated CDF and the field strength of the experimental CDF for $P=10^{-1}$.

\begin{tabular}{|c|c|c|c|c|c|}
\hline Distributions & Rayleigh & Nakagami- $m$ & Weibull & Rice & $\alpha-\mu$ \\
\hline \multicolumn{6}{|c|}{ Total measurements } \\
\hline Mean (dB) & -2.83 & 0.02 & 0.06 & 0.28 & 0.16 \\
\hline $\mathrm{rms}(\mathrm{dB})$ & 3.47 & 0.44 & 0.42 & 0.68 & 0.48 \\
\hline \multicolumn{6}{|l|}{ Route \#1 } \\
\hline Mean (dB) & -2.87 & 0.03 & 0.09 & 0.26 & 0.18 \\
\hline $\operatorname{rms}(\mathrm{dB})$ & 3.36 & 0.40 & 0.38 & 0.57 & 0.45 \\
\hline \multicolumn{6}{|l|}{ Route \#2 } \\
\hline Mean $(\mathrm{dB})$ & -2.36 & 0.08 & 0.10 & 0.29 & 0.22 \\
\hline $\mathrm{rms}(\mathrm{dB})$ & 2.95 & 0.39 & 0.39 & 0.70 & 0.54 \\
\hline \multicolumn{6}{|l|}{ Route \#3 } \\
\hline Mean $(\mathrm{dB})$ & -2.31 & 0.05 & 0.12 & 0.43 & 0.22 \\
\hline $\mathrm{rms}(\mathrm{dB})$ & 3.00 & 0.52 & 0.51 & 0.89 & 0.57 \\
\hline \multicolumn{6}{|l|}{ Route \#4 } \\
\hline Mean $(\mathrm{dB})$ & -2.95 & -0.17 & -0.02 & 0.25 & 0.12 \\
\hline $\mathrm{rms}(\mathrm{dB})$ & 3.64 & 0.53 & 0.47 & 0.63 & 0.48 \\
\hline \multicolumn{6}{|l|}{ Route \#5 } \\
\hline Mean (dB) & -3.79 & -0.24 & -0.08 & 0.14 & 0.06 \\
\hline $\mathrm{rms}(\mathrm{dB})$ & 4.29 & 0.48 & 0.39 & 0.62 & 0.34 \\
\hline \multicolumn{6}{|l|}{ Route \#6 } \\
\hline Mean (dB) & -3.55 & -0.05 & 0.02 & 0.13 & 0.1 \\
\hline $\operatorname{rms}(\mathrm{dB})$ & 4.09 & 0.33 & 0.29 & 0.46 & 0.37 \\
\hline \multicolumn{6}{|l|}{ Route \#7 } \\
\hline Mean (dB) & -2.94 & -0.12 & -0.00 & 0.18 & 0.11 \\
\hline $\operatorname{rms}(\mathrm{dB})$ & 3.56 & 0.42 & 0.37 & 0.47 & 0.37 \\
\hline \multicolumn{6}{|l|}{ Route \#8 } \\
\hline Mean $(\mathrm{dB})$ & -3.32 & -0.1 & -0.02 & 0.24 & 0.08 \\
\hline $\mathrm{rms}(\mathrm{dB})$ & 4.25 & 0.38 & 0.34 & 0.61 & 0.42 \\
\hline
\end{tabular}

\section{Conclusions}

In this work, an extensive analysis of the small-scale fading distribution in $\mathrm{V} 2 \mathrm{~V}$ channels has been performed. The analysis is based on a narrowband measurement campaign carried out in both highway and urban environments. The parameters of the Rayleigh, Rice, Weibull, Nakagami- $m$, and $\alpha-\mu$ distributions have been estimated from the measurements by statistical inference. The goodness-of-fit of the theoretical distributions to the empirical distribution has been evaluated using the K-S statistical test. In all the highway routes assessed, the mean of the Rician $K$-parameter exceeds $10 \mathrm{~dB}$, which corresponds to situations with dominant MPCs. On the contrary, the results show that the mean of the Rician $K$-parameter ranges from 4.21 to $8.82 \mathrm{~dB}$ in urban environments.

The results derived from the application of the K-S test have shown that the Nakagami-m, Rice, Weibull, and $\alpha-\mu$ distributions can match satisfactorily the empirical distribution associated with the measurements. Nevertheless, the $\alpha-\mu$ distribution exhibits a better fit compared to the 
other distributions, making its use interesting to model the small-scale fading in V2V channels, where the large channel variations due to the mobility of $\mathrm{Tx}$ and $\mathrm{Rx}$ terminals, together with the other interacting objects, make it difficult to separate both the small- and large-scale fading. It is also worth noting that the Weibull distribution provides the best match for the lower tails of the empirical distribution.

\section{Competing Interests}

The authors declare that they have no competing interests.

\section{Acknowledgments}

This work has been funded in part by the Programa Estatal de Fomento de la Investigación Científica y Técnica de Excelencia del Ministerio de Economía y Competitividad, Spain, TEC2013-47360-C3-3-P, and the Departamento Administrativo de Ciencia, Tecnología e Innovación COLCIENCIAS en Colombia.

\section{References}

[1] A. F. Molisch, F. Tufvesson, J. Karedal, and C. F. Mecklenbräuker, "A survey on vehicle-to-vehicle propagation channels," IEEE Wireless Communications, vol. 16, no. 6, pp. 12-22, 2009.

[2] I. Sen and D. W. Matolak, "Vehicle-vehicle channel models for the 5-GHz band," IEEE Transactions on Intelligent Transportation Systems, vol. 9, no. 2, pp. 235-245, 2008.

[3] G. Acosta-Marum and M. A. Ingram, "Six time- and frequencyselective empirical channel models for vehicular wireless LANs," IEEE Vehicular Technology Magazine, vol. 2, no. 4, pp. 4-11, 2007.

[4] C.-X. Wang, X. Cheng, and D. Laurenson, "Vehicle-to-vehicle channel modeling and measurements: recent advances and future challenges," IEEE Communications Magazine, vol. 47, no. 11, pp. 96-103, 2009.

[5] R. He, O. Renaudin, V.-M. Kolmonen et al., "Characterization of quasi-stationarity regions for vehicle-to-vehicle radio channels," IEEE Transactions on Antennas and Propagation, vol. 63, no. 5, pp. 2237-2251, 2015.

[6] N. Akhtar, S. C. Ergen, and O. Ozkasap, "Vehicle mobility and communication channel models for realistic and efficient highway VANET simulation," IEEE Transactions on Vehicular Technology, vol. 64, no. 1, pp. 248-262, 2015.

[7] H. Fernández, L. Rubio, J. Reig, V. M. Rodrigo-Peñarrocha, and A. Valero, "Path loss modeling for vehicular system performance and communication protocols evaluation," Mobile Networks and Applications, vol. 18, no. 6, pp. 755-765, 2013.

[8] J. Maurer, T. Fügen, and W. Wiesbeck, "Narrow-band measurement and analysis of the inter-vehicle transmission channel at $5.2 \mathrm{GHz}$,' in Proceedings of the 55th Vehicular Technology Conference, pp. 1274-1278, May 2002.

[9] L. Bernado, T. Zemen, F. Tufvesson, A. F. Molisch, and C. F. Mecklenbräuker, "Time- and frequency-varying $K$-factor of non-stationary vehicular channels for safety-relevant scenarios," IEEE Transactions on Intelligent Transportation Systems, vol. 16, no. 2, pp. 1007-1017, 2015.
[10] O. Renaudin, V.-M. Kolmonen, P. Vainikainen, and C. Oestges, "Non-stationary narrowband MIMO inter-vehicle channel characterization in the $5-\mathrm{GHz}$ band," IEEE Transactions on Vehicular Technology, vol. 59, no. 4, pp. 2007-2015, 2010.

[11] J. Yin, G. Holland, T. Elbatt, F. Bai, and H. Krishnan, "DSRC channel fading analysis from empirical measurement," in Proceedings of the 1st International Conference on Communications and Networking in China (ChinaCom '06), pp. 1-5, Beijing, China, October 2006.

[12] L. Cheng, B. E. Henty, D. D. Stancil, F. Bai, and P. Mudalige, "Mobile vehicle-to-vehicle narrow-band channel measurement and characterization of the $5.9 \mathrm{GHz}$ dedicated short range communication (DSRC) frequency band," IEEE Journal on Selected Areas in Communications, vol. 25, no. 8, pp. 1501-1516, 2007.

[13] R. He, A. F. Molisch, F. Tufvesson, Z. Zhong, B. Ai, and T. Zhang, "Vehicle-to-vehicle propagation models with large vehicle obstructions," IEEE Transactions on Intelligent Transportation Systems, vol. 15, no. 5, pp. 2237-2248, 2014.

[14] D. W. Matolak and J. Frolik, "Worse-than-rayleigh fading: experimental results and theoretical models," IEEE Communications Magazine, vol. 49, no. 4, pp. 140-146, 2011.

[15] X. Cheng, C.-X. Wang, B. Ai, and H. Aggoune, "Envelope level crossing rate and average fade duration of nonisotropic vehicle-to-vehicle ricean fading channels," IEEE Transactions on Intelligent Transportation Systems, vol. 15, no. 1, pp. 62-72, 2014.

[16] H. Fernández, L. Rubio, V. M. Rodrigo-Penarrocha, and J. Reig, "Path loss characterization for vehicular communications at $700 \mathrm{MHz}$ and $5.9 \mathrm{GHz}$ under LOS and NLOS conditions," IEEE Antennas and Wireless Propagation Letters, vol. 13, pp. 931-934, 2014.

[17] M. D. Yacoub, “The $\alpha-\mu$ distribution: a physical fading model for the Stacy distribution," IEEE Transactions on Vehicular Technology, vol. 56, no. 1, pp. 122-124, 2007.

[18] F. J. Massey Jr., "The Kolmogorov-Smirnov test for goodness of fit," Journal of the American Statistical Association, vol. 46, no. 253, pp. 68-78, 1951.

[19] J. Reig, M.-T. Martínez-Inglés, L. Rubio, V.-M. RodrigoPeñarrocha, and J. Molina-García-Pardo, "Fading evaluation in the $60 \mathrm{GHz}$ band in line-of-sight conditions," International Journal of Antennas and Propagation, vol. 2014, Article ID 984102, 12 pages, 2014.

[20] N. L. Johnson, S. Kotz, and N. Balakrishnan, Continuous Univariate Probability Distributions, vol. 1, John Wiley \& Sons, New York, NY, USA, 2nd edition, 1994.

[21] NIST, "1.3.5.11. measures of skewness and kurtosis," February 2016, http://www.itl.nist.gov/div898/handbook/eda/section3/ eda35b.htm.

[22] A. F. Molisch, Wireless Communications, John Wiley \& Sons, 3rd edition, 2010. 

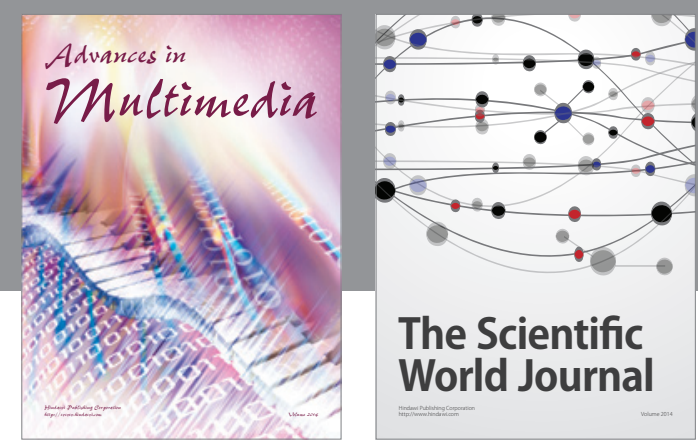

The Scientific World Journal
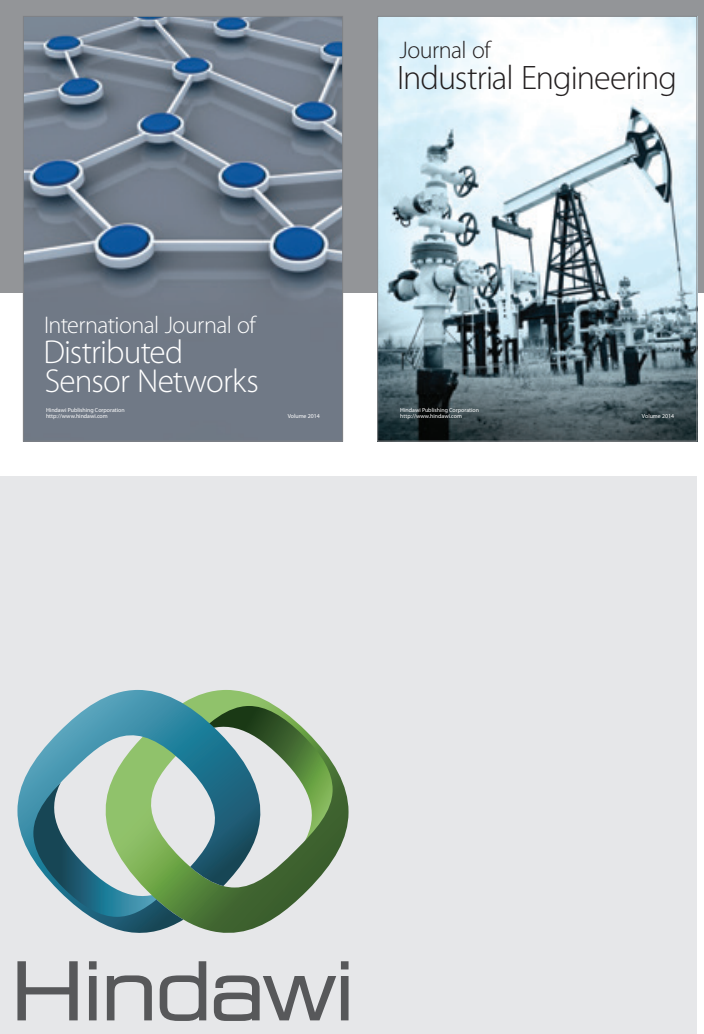

Submit your manuscripts at

http://www.hindawi.com

\section{Computer Networks} and Communications
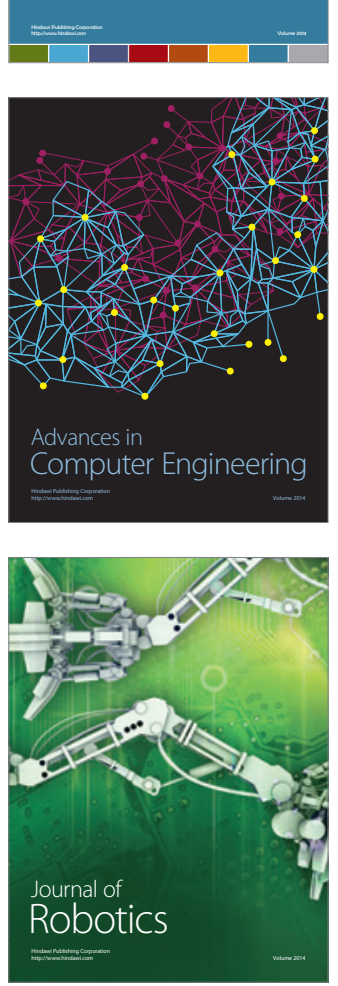
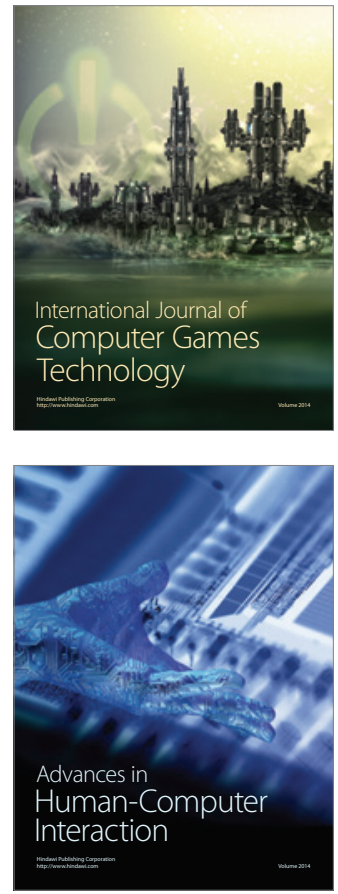
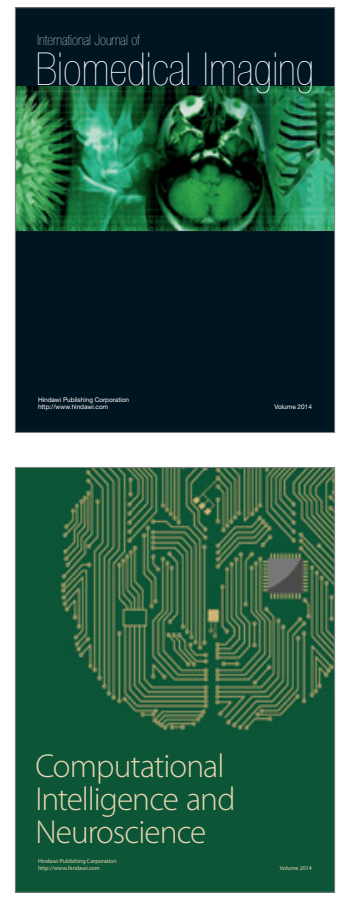
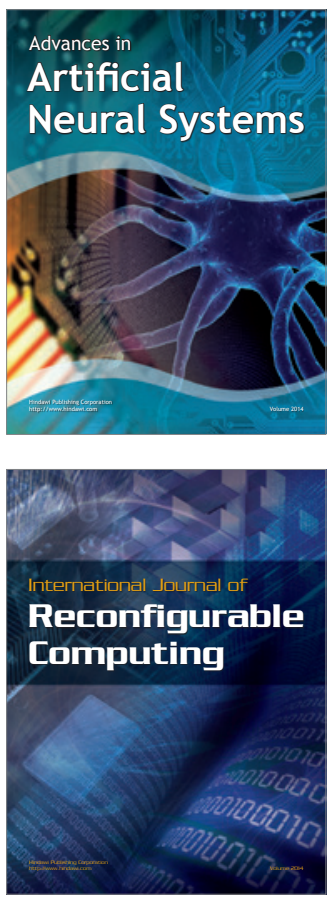
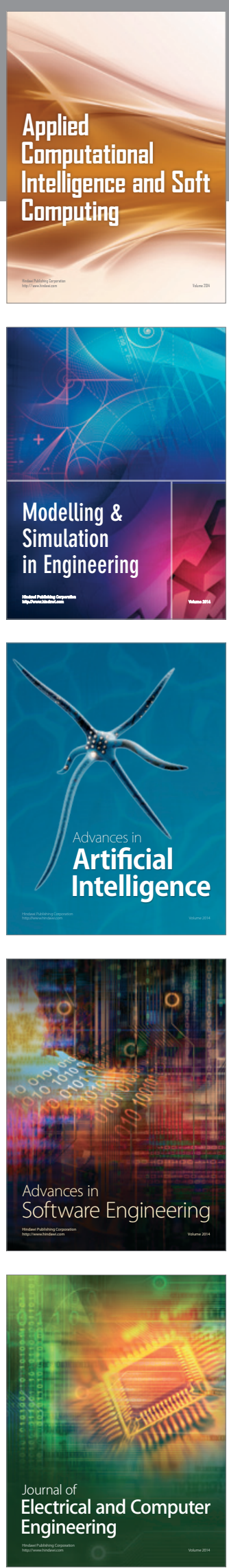